\title{
Efficiency and Productivity Assessment of Wind Farms
}

\author{
Clara Bento Vaz and Ângela Paula Ferreira
}

\begin{abstract}
This study develops a framework to provide insights regarding the performance of the farms of an energy player in the Portuguese wind sector. The focus of the wind farm performance assessment is on the operating stage which corresponds to the electrical energy generation process, during 2010 and 2011. In a first stage, Data Envelopment Analysis is used to measure the efficiency of wind farms in generating electrical energy from the resources available and nondiscretionary variables. This analysis enables the identification of the best practices of the efficient farms which can be emulated by inefficient ones. In a second stage, changes in wind farms productivity are investigated using Malmquist index. Bootstrap procedures are applied to obtain statistical inference on the efficiency estimates. We conclude that almost all farms decreased overall productivity levels, mainly due to the decline in the productivity levels of the frontier, which is in accordance with the decrease in wind availability observed in 2011.
\end{abstract}

\section{Introduction}

Data Envelopment Analysis (DEA) has been accepted as an important approach for performance assessment and benchmarking in several sectors [21]. Studies using DEA to assess the performance of wind farms are still scarce [12].

C.B. Vaz $(\square)$

Polytechnic Institute of Bragança, Campus de Santa Apolónia, Apartado 1134, 5301-857,

Bragança, Portugal

CGEI / INESC TEC - INESC Technology and Science, Porto, Portugal

e-mail: clvaz@ipb.pt

Â.P. Ferreira

Polytechnic Institute of Bragança, Campus de Santa Apolónia, Apartado 1134, 5301-857, Bragança, Portugal

CISE - Electromechatronic Systems Research Centre, University of Beira Interior, Covilhã, Portugal

e-mail: apf@ipb.pt

(C) Springer International Publishing Switzerland 2015

J.P. Almeida et al. (eds.), Operational Research, CIM Series in Mathematical

Sciences 4, DOI 10.1007/978-3-319-20328-7_22 
In a Policies Scenario taking into account both existing policies and declared intentions by countries, world primary energy demand is projected to increase by $1.2 \%$ per year, on average, between the current year and 2035. Electricity demand is projected to grow by a higher rate, $2.2 \%$ per year, given that it is expected that applications, formerly based on chemical energy, will be based on electrical energy in the following decades [13]. In order to cope with the worldwide climate changes, policies are being implemented to enhance the transition toward low-carbon technologies in the power sector. In this context, the share of world electricity generation from renewable sources is projected to grow, whereas the wind energy is the most representative source. According to Global Wind Energy Council (GWEC), installed wind capacity has grown to an accumulative worldwide level of $318 \mathrm{GW}$ from which $35.5 \mathrm{GW}$ had been installed in 2013. Europe is still the largest wind energy generator, despite the fact that other markets (e.g. USA, India, China) have also launched in recent years. Portugal accounts for about four percent of the wind energy installed capacity of the European Union, with approximately $4.7 \mathrm{GW}$ of accumulated installed capacity in 2013 which is capable to generate about $20 \%$ of electricity consumption [6].

Several factors contributed to the development of the wind energy sector in Portugal. Since 2002, the implementation of a legal stable framework by the Portuguese government and several financial support programs implemented by the European Commission have promoted the penetration of electricity generated from renewable energy sources [14]. Despite technology potential and investments in a low-carbon energy market, the progress is too slow on attending outlined targets. The main reasons for the slow progress are related with a low share of energy-related investment in R\&D activities, high investments when compared with thermal based electricity, uncertain time for the return of the capital invested, technical limitations of power systems in supporting large penetration from variable renewable energy and environmental impact.

There are ten main wind farm promoters acting in the wind energy sector in Portugal, with farms connected to the transmission or distribution grid system. Each promoter is concerned, besides appropriate financial management, to ensure the maximum energy generation, with the highest availability rates and cost-effectiveness in terms of operation and maintenance. In this context, the development of performance assessment methodologies in the portfolio of a given promoter allows the identification of wind farms with the best practices in the operating stage in order to be emulated by inefficient farms. The use of DEA can contribute to enhance those methods through assessment of the potential for efficiency improvements and exploring their productivity change over time, considering the emergent interest on productivity growth in electrical utilities. This is explored by using Malmquist index which can be decomposed in efficiency change and technological change. The efficiency change can be associated with internal operating practices observed in each farm, while the technological change can be related to specific conditions in which farms have to operate, for instance, the level of wind availability in each year. 
This study proposes a framework based on DEA to provide insights regarding the performance of the farms of an energy player in the Portuguese wind sector. In a first stage, DEA is used to measure the operating efficiency of the wind farms and to identify the benchmarks, followed by a second stage, were changes in wind farms productivity are investigated using the Malmquist index over 2 years, in which the wind energy sector suffered a considerable decrease in the electric energy generated. The robustness of the scores achieved by DEA models can be tested by using bootstrapping methods $[17,18]$. The proposed framework is applied to a case study, giving insights into the performance assessment of wind farms from Iberwind which has a market share of $18 \%$ on the Portuguese wind energy sector.

This study is organized as follows: next section points out a literature survey about performance assessment of wind farms, Sect. 3 presents the methodology to assess the efficiency and productivity of wind farms, Sect. 4 characterizes the context setting in the wind energy sector and applies the methodology to the case study, and finally Sect. 5 rounds up the paper with the main conclusions.

\section{Literature Review}

Zhou et al.[21] presented a survey on DEA energy sector and environmental modeling, from which benchmarking of electricity utilities accounted for the large number of studies although it did not include any application in wind energy sector. Regarding the methodology, this study pointed out that the constant returns to scale reference technology and the radial efficiency measures are still the most widely used specifications and there has been a growing interest on the use of Malmquist index to assess the productivity change over time.

The works performed by Iglesias et al. [12] and Pestana and Barros [16] focus on the efficiency assessment of wind farms and argue the importance to model the non-discretionary factors such as the wind speed and its availability in each farm.

Iglesias et al. [12] used DEA and Stochastic Frontier Analysis (SFA) methodologies to measure the efficiency of a group of wind farms located in Spain. Models are output oriented concerning the generated energy, based on a relationship between capital, labor and fuel, similar to a conventional energy conversion system. Capital factor is evaluated by the installed capacity in each farm and labor factor considers the number of fulltime employers responsible for operation, control and maintenance of the farms. Concerning fuel, this input is estimated based on the wind power incident per unit time on the interposed surface of the wind turbines and the annual average wind speed at each site.

Pestana and Barros [16] used SFA and stochastic production econometric frontier to assess efficiency of Portuguese wind farms from different promoters. Outputs are measured by generated energy and capacity utilization, and the inputs are price for labor and capital invested proxied by the book value of physical assets. Findings of this study are that Portuguese wind farms' operational activity is affected by heterogeneous factors such as farm size, managerial practices and ownership. 
This paper improves the existing methodologies in performance assessment of wind farms from a given promoter to provide additional insights into efficiency and productivity growth over time by exploring the benchmarking analysis and Malmquist index. To increase the robustness of the efficiency and productivity results achieved, the bootstrapping framework [17, 18] is used. These methodological aspects, which have not been used in previous studies, allow a better understanding of wind farms during operating stage which can support the decision maker in benchmarking the wind farms in repowering or overpowering processes.

\section{Performance Assessment Methodology}

The methodology proposed in this study intends to explore the productivity and the efficiency of wind farms. In a first stage, DEA is used to assess the farms efficiency by taking into account the resources and the non-discretionary variable, the wind, available in each farm to generate electric energy. This approach enables benchmarking among farms. The robustness of efficiency scores is tested by using bootstrap framework [17]. In a second stage, we use panel data to assess the overall productivity change over time of the farms by using the Malmquist index and its components [9], efficiency change and technological change. The efficiency change measures if the farm is moving closer or farther from the frontier while the technological change measures shifts in the frontier that can be characterized by progression, regression or both. Finally, the robustness of these indexes is tested by using bootstrapping [18] which allows the identification of significant aspects that may explain the performance of each farm over time. The following sections present the proposed methodology in detail.

\subsection{DEA Model}

DEA is a non-parametric technique to assess the relative efficiency of an homogeneous set of Decision Making Units (DMUs) in producing multiple outputs from multiple inputs. This allows to identify the "best practices DMUs" and their linear combination defines the frontier technology. By reference to this frontier, a single summary measure of efficiency is calculated for each DMU. In the original DEA model proposed by [4], the efficiency score of each DMU is estimated by using the frontier technology characterized by Constant Returns to Scale (CRS). For an output oriented analysis, we consider a technology involving $n$ production units defined by $j(j=1, \ldots, n)$, which use the inputs $x_{i j}\left(x_{1 j}, \ldots, x_{m j}\right) \in R_{+}^{m}$, to obtain the outputs $y_{r j}\left(y_{1 j}, \ldots, y_{s j}\right) \in R_{+}^{s}$, i.e., the production possibility set (PPS). In this model, the efficiency of each DMU $j_{o}$ is given by the reciprocal of the factor $(\theta)$ by which the 
outputs of the DMU $j_{o}$ can be expanded, according to the following linear model:

$$
\begin{gathered}
\max \left\{h_{j_{o}}=\theta \mid x_{i j_{o}} \geq \sum_{j=1}^{n} \lambda_{j} x_{i j}, \quad i=1, \ldots, m\right. \\
\theta y_{r j_{o}} \leq \sum_{j=1}^{n} \lambda_{j} y_{r j}, \quad r=1, \ldots, s \\
\left.\lambda_{j} \geq 0, \quad \forall_{j}\right\}
\end{gathered}
$$

Model (1) assesses the relative efficiency of DMUs in the achievement of the output levels given the resources used. The measure of efficiency, given by $1 / \theta^{*}$, equals to $100 \%$ when the unit under assessment is efficient, whereas lower scores indicate the existence of inefficiencies. For the inefficient units there is evidence that it is possible to obtain higher levels of outputs with the same or lower levels of the inputs currently used. For these units, it is also possible to obtain, as by-products of the DEA efficiency assessment, a set of targets for becoming efficient. The input and output targets for a DMU $j_{o}$ under assessment are obtained as follows:

$$
\begin{gathered}
x_{i j_{o}}^{o}=x_{i j_{o}}-s_{i}^{*}=\sum_{j=1}^{n} \lambda_{j}^{*} x_{i j} \\
y_{r j_{o}}^{o}=\theta_{o}^{*} y_{r j_{o}}+s_{r}^{*}=\sum_{j=1}^{n} \lambda_{j}^{*} y_{r j}
\end{gathered}
$$

where the variables $s_{i}^{*}$ and $s_{r}^{*}$ are the slacks corresponding to the input $i$ and output $r$ constraints, respectively, given by the optimal solution of model (1). The benchmarks for the inefficient DMUs $j_{o}$ are the units with values of $\lambda_{j}^{*}>0$ in the optimal solution of model (1). These are the Pareto-efficient DMUs which have $\theta_{o}^{*}=1$ and all slacks are equal to zero.

Model (1) enables to assess the Technical Efficiency (TE) for each DMU which can be due to the ineffective operation of the production process in transforming inputs into outputs and also due to the divergence of the entity from the Most Productive Scale Size (MPSS), considering the most productive frontier characterized by constant returns to scale. If a DMU has TE equals to 1 , it is efficient in transforming inputs into outputs and it will have MPSS, by operating at optimal scale size. Banker et al. [1] proposed the DEA model that assesses the Pure Technical Efficiency (PTE) for each DMU by using the frontier characterized by Variable Returns to Scale (VRS) which is achieved by including the constraint $\sum_{j=1}^{n} \lambda_{j}=1$ in model (1). The Pure Technical Efficiency (PTE) for each DMU enables to measure the inefficiency due to the ineffective operation of the production process in transforming inputs into outputs. The scale efficiency (SE) is measured by the distance between CRS and VRS frontiers which corresponds to the divergence 
of the DMU from the MPSS and is given by the ratio $\frac{T E}{P T E}$. Thus, the Technical Efficiency (TE) is decomposed in pure technical efficiency and scale efficiency components.

For Pareto-efficient DMUs, in VRS frontier, it is possible to identify the local Returns to Scale (RTS) which enables to identify advantages in changing the scale of DMUs. In the study case under analysis, this information is very useful in repowering processes of wind farms. If increasing returns to scale hold at a Paretoefficient DMU, then increasing its input levels by a given percentage will lead to expansion of its output levels by a larger percentage, i.e., the scale size of the DMU should be increased. If a DMU is operating at a point where decreasing returns to scale hold, it should decrease its scale size. If a DMU operates at constant returns to scale point, its scale size is considered optimal. The approach proposed in [8] is used to characterize the RTS of Pareto-efficient wind farms.

It is well known that DEA results are sensitive to sample variation which leads to deviation around the observed frontier. To overcome this uncertainty, we used bootstrapping to obtain unbiased estimates. Bootstrapping was first introduced by [7] and it is based on the idea of resampling from the given sample of observations to replicate datasets from which we can make the statistical inference. The bootstrapping approach proposed by [17] is appropriated to use with the DEA efficiency estimates which range from zero to one. For each DMU, the $\theta$ derived from model (1) is corrected for the bias to derive the bias-corrected score $\hat{\hat{\theta}}$ and the confidence interval. These scores are used to assess the wind farms performance. This procedure was implemented using the statistical software $\mathrm{R}$ including the FEAR library, developed by [20].

\subsection{Malmquist Index on Evaluation of Overall Productivity}

In energy sectors, it is of great interest the investigation of productivity change over time [21]. The Malmquist productivity index was introduced by [3] and developed further in the context of performance assessments by [9] to accomplish performance comparisons of DMUs over time. The high popularity of this method is related with several factors. Firstly, it is not necessary to use price data, assumptions of cost minimization or revenue maximization. Secondly, it can be used either in oriented or non-oriented analysis. Thirdly, it enables the determination of the total factor productivity in the generic case where production technology uses multiple inputs to produce multiple outputs by deriving efficiency scores in DEA models. Fourthly, the index is applied to the measurement of productivity change over time, and can be decomposed into an efficiency change index and a technological change index. These indexes are investigated in the case study between both years, since the wind availability decreases in 2011. It is important to know what happens with the frontier, which is captured by technological change index. In these adverse conditions, it is important to identify the behavior of each farm in catching up the 
frontier, i.e., if it is getting closer or farther from the frontier, which is captured by efficiency change index.

The Malmquist index, as proposed by [9], is used to derive the overall productivity of each DMU. It is based on radial measures which are defined by distance functions. In output-oriented analysis, the output distance function is equal to the efficiency score estimated by model (1), given by $1 / \theta^{*}$ for each DMU for a given period. Consider a set of $n$ DMUs in period $t$, which use the inputs $x^{t} \in R_{+}^{m}$ to obtain the outputs $y^{t} \in R_{+}^{s}$, and the same $n$ DMUs in period $t+1$, which use the inputs $x^{t+1} \in R_{+}^{m}$ to obtain the outputs $y^{t+1} \in R_{+}^{s}$. To simplify the notation, the efficiency score estimated for each $\mathrm{DMU}_{j_{o}}$ in period $t$ is given by $E_{o}^{t}(t)$ while the efficiency score estimated for each DMU in period $t+1$ is given by $E_{o}^{t+1}(t+1)$. Thus, the score in parenthesis represents the period in each DMU is assessed while the superscript denotes the frontier technology used as reference. The Malmquist index derived for each DMU is obtained as:

$$
I_{o}^{t+1, t}=\left(\frac{E_{o}^{t}(t+1)}{E_{o}^{t}(t)} \frac{E_{o}^{t+1}(t+1)}{E_{o}^{t+1}(t)}\right)^{\frac{1}{2}}
$$

In terms of interpretation, a score of $I_{o}^{t+1, t}>1$ indicates better performance in period $t+1$ than in period $t$.

The mixed-period distance functions, $E_{o}^{t}(t+1)$ and $E_{o}^{t+1}(t)$, can be greater, equal or lower than 1. For example, the distance function derived to the period $t+1$ for a DMU observed in period $t$ can be lower or equal to 1 if the input-output vector of this DMU belongs to the PPS of period $t+1$. This occurs for $E_{o}^{t}(t)$ and $E_{o}^{t+1}(t+1)$ cases. In opposite, the distance function derived to the period $t+1$ for a DMU observed in period $t$ is higher than 1 , if the input-output vector of this DMU is outside the PPS of the period $t+1$.

According to [9], this index can be decomposed in two components: $I E_{o}^{t+1, t}$ and $I F_{o}^{t+1, t}$. The sub-index $I E_{o}^{t+1, t}$ corresponds to efficiency change and compares the efficiency spread between the periods observed for each DMU. The sub-index $I F_{o}^{t+1, t}$ corresponds to technological change and compares the relative position of the frontiers associated to periods $t$ and $t+1$ for the input-output mix of each DMU observed. This decomposition implies that the sources of better performance can be associated with two factors: less dispersion in the efficiency score of DMU in each period and/or better productivity associated to the period frontier.

The efficiency change derived for each DMU is calculated according to:

$$
I E_{o}^{t+1, t}=\frac{E_{o}^{t+1}(t+1)}{E_{o}^{t}(t)}
$$

A value of $I E_{o}^{t+1, t}>1$ means that the efficiency spread is smaller in DMU observed in period $t+1$ than the one observed in period $t$, measuring how much the DMU is getting closer (i.e. catching up) or farther from the frontier. 
Concerning the technological change derived for each DMU, it is given by:

$$
I F_{o}^{t+1, t}=\left(\frac{E_{o}^{t}(t)}{E_{o}^{t+1}(t)} \frac{E_{o}^{t}(t+1)}{E_{o}^{t+1}(t+1)}\right)^{\frac{1}{2}}
$$

When $I F_{o}^{t+1, t}$ is higher than 1, this means that the productivity of frontier $t+1$ is better than the productivity of frontier $t$, which implies that the frontier has progressed. This index can be seen as an average aggregated change in technology of a DMU since it is obtained as the geometric mean of two components. The first component $\left(\frac{E_{o}^{t}(t)}{E_{o}^{t+1}(t)}\right)$ corresponds to the distances between the frontiers $t$ and $t+1$ when assessed for the DMU observed in period $t$. The second component $\left(\frac{E_{o}^{t}(t+1)}{E_{o}^{t+1}(t+1)}\right)$ is calculated in a similar way for the same DMU observed in period $t+1$.

It is possible to analyze globally the relative position of the two frontiers, which enables to identify if the frontiers have regressed, progressed or crossed over. To do so, it is necessary to analyze each component of $I F_{o}^{t+1, t}$ for all DMUs observed in the periods under analysis. Some typical situations may occur: for instance, if the component is always higher than 1 , this means that there has been a progression in the technology; on the other hand, if the component is always lower than 1, this means that there has been a regression in the technology; in the case when there is at least one component higher than 1 and one component lower than 1, this indicates that frontiers are crossed over, signifying that for some input-output mix the frontier progressed and for others, the frontier regressed.

The bootstrapping framework proposed by [18] is used to evaluate the robustness of the estimates of $I_{o}^{t+1, t}, I E_{o}^{t+1, t}$ and $I F_{o}^{t+1, t}$ (hereinafter $I, I E$ and $I F$, respectively) obtained for each DMU, which allows the computation of confidences intervals for each index. If the interval contains the value 1 , we cannot infer that significant changes occurred in the corresponding DMU. On the other hand, if the lower and upper bounds are smaller (or higher) than 1, this implies that there was a decline (or progress) in the DMU. This approach is currently used in several studies $[10,11,15,19]$. This analysis is extended to the components of $I F$ for all DMUs observed to find out the relative position of the frontiers.

\section{Performance Assessment of Wind Farms}

This section applies the methodology proposed in previous section to evaluate the performance of wind farms owned by Iberwind in Portugal. This study focus in the wind farms efficiency analysis during operating stage, for a given distribution of wind speed in the geographical location of the farms, installed capacity and number of wind turbines, oriented to the maximization of the output electric energy generated. The rationale for this context is presented in the following sub-section. 


\subsection{Contextual Setting}

Relevant decisions and factors that affect the productivity of wind farms are prior to start-up, including for instance wind farm location and layout design, engineering design process such as the installed capacity, type of generator, turbine aerodynamics and active control system. This work focuses on the performance assessment of wind farms in the operating phase, i.e., when they perform the energy conversion and it is delivered to the utility grid. Even though the performance of a wind farm is closely linked to prior start-up phase, the operating phase is relevant throughout estimated lifetime of the assets, from the point of view of maximizing the energy generation, ensuring the highest availability rates and cost-effective operation and maintenance schemes.

Wind is a variable source of power: output rises and falls as wind strength fluctuates in a hourly or $10 \mathrm{~min}$ time scale, although, its variability is consistent from year to year. Wind speeds suitable for electricity generation range from approximately $5 \mathrm{~m} / \mathrm{s}$ (cut in speed) to 25 or $30 \mathrm{~m} / \mathrm{s}$ (cut out speed). The frequency of wind speeds usually fits a Weibull distribution and an average value, for itself, does not translate the amount of energy that a wind farm can produce.

Installed capacity and the number of wind turbines in a farm, along with the variability of wind, relate to the capacity factor of a wind farm, i.e., the ratio of actual productivity in a year to its theoretical maximum. The rated power of a unit of the wind farm (given by the ratio of the installed capacity and the number of wind turbines) if small, can lead to an higher capacity factor of the farm, and, consequently, it may not be able to produce energy at higher wind speeds, which translates in less profit. On the other hand, if the rated power of each turbine is high, it may stall at low wind speeds and the extra power at high wind speeds they are able to convert, may not compensate the higher costs of the turbine. Therefore, these resources are important to assess efficiency and productivity analysis during operating phase and may provide useful information in repowering or overpowering processes.

Concerning the output, it should be point out that electric energy generated from wind is not constrained by load demand or other market players, as currently regulated.

\subsection{DEA Model}

Each DMU is a wind farm which is formed by a group of wind turbines connected to the transmission or distribution grid utility. The number of DMUs under analysis is 31, spread out in North and Center of Portugal. The final data set considers 30 wind farms since one of them was eliminated due to a repowering process that began in 2010. Total capacity installed ascends to $683.75 \mathrm{MW}$ through 319 wind turbines, from 15 different models, provided by five manufacturers (Vestas, Nordex, Enercon, 
GE and WinWind). We consider a panel data set collected from Annual Reports and Accounts, for 2010 and 2011 years. ${ }^{1}$ The 30 farms under analysis are located in six wind typical geographical locations in Portugal (Bragança, Vila Real, Viseu, Coimbra, Leiria and Lisboa).

The wind farms can be considered homogenous as they result from similar setup stages and use a similar generation process. The output-oriented perspective is used, as the objective of the farms is to produce maximum electric energy, taking into account the non-discretionary variable, the wind, and the resources available in each farm. For each assessed farm, the output-oriented model seeks feasible input and output levels which have the following properties: the outputs are the maximum multiple of the outputs observed in the assessed farm and the inputs are no higher than those of the assessed farm. Thus, for an inefficient farm there is evidence that it is possible to increase the level of the electrical energy produced by following the best practices observed in the benchmarks (efficient farms). These best practices can be associated with the management of the resources in each farm related to the planning of the maintenance schemes for periods in time when the wind is not suitable for producing electrical energy. Thus, the model enables to identify the inefficient farms where there is evidence that they can improve the management of their controllable resources to catch the highest level of the wind hours and, consequently, can increase the electrical energy produced.

The CRS frontier is used to assess the technical efficiency of wind farms observed. In order to model the farm activity, the input-output set should cover the full range of resources used and the outputs that are relevant for the objectives of the analysis [5]. Thus, the output corresponds to the amount of electric energy delivered to the grid and the inputs considered are the installed power, number of turbines and wind availability. The descriptive measures concerning the inputs and output under analysis are summarized in Table 1 . Installed power capacity of the farms is determined by the number of wind turbines multiplied by the rated power of each one. The number of turbines relates with the area occupied by the farm. To capture the effect of the wind variability into the model, we consider the number of hours per year that wind speed is within the range defined by cut in and cut out speeds (hereinafter named wind hours). For each wind farm location, the wind data is collected from a meteorological data base throughout identification of the station which represents its wind profile, defined by the nearest meteorological station. The inclusion of this non-discretionary input assures that a farm with unfavorable conditions regarding wind resource is not penalized in the performance assessment. The wind hours is an internal non-discretionary input which should be used for the definition of the PPS, according to [2]. Data concerning the maintenance schemes and operation costs are confidential and, consequently, they are not included in the model.

${ }^{1}$ The constraint of the panel data is limited to 2010 and 2011, because there is no available wind data from meteorological stations in former years and also in recent years. 
Table 1 Mean and standard deviation values for inputs and output of wind farms

\begin{tabular}{|c|c|c|c|c|}
\hline & \multicolumn{2}{|l|}{2010} & \multicolumn{2}{|l|}{2011} \\
\hline & Mean & Sta. Dev. & Mean & Sta. Dev. \\
\hline \multicolumn{5}{|l|}{ Inputs } \\
\hline Installed power (MW) & 22.4 & 30.4 & 22.4 & 30.4 \\
\hline No of wind turbines & 10.4 & 10.7 & 10.4 & 10.7 \\
\hline Wind hours & 3773.6 & 1082.4 & 3280.5 & 980.4 \\
\hline \multicolumn{5}{|l|}{ Output } \\
\hline Electric Energy (GWh) & 56.5 & 81.5 & 51.1 & 75.8 \\
\hline
\end{tabular}

Table 2 Summary results of original and bootstrapped efficiency scores

\begin{tabular}{l|l|l|l|l|r|l}
\hline \multirow{2}{*}{} & \multicolumn{2}{|l|}{ Year 2010 } & \multicolumn{3}{l}{ Year 2011 } \\
\cline { 2 - 7 } & Bias cor. eff. (\%) & Bias (\%) & Eff. est. (\%) & Bias cor. eff. (\%) & Bias (\%) & Eff. est. (\%) \\
\hline Mean & 72.92 & -8.00 & 77.73 & 66.33 & -10.54 & 73.97 \\
\hline Sta. Dev. & 10.13 & 4.30 & 12.33 & 10.86 & 4.92 & 13.44 \\
\hline
\end{tabular}

The standard deviation of observed variables is quite high compared with the mean values, indicating a considerable amount of diversity in the wind farms.

The summary of the technical efficiency estimates, using the formulation shown in model (1) are presented in Table 2. The robustness of these estimates is tested by calculating the bias-corrected efficiency scores (as the inverse of $\hat{\hat{\theta}}$ ) [17] which summary results are also presented in Table 2.

The efficiency estimated scores are relative, since the farms in a given year are only compared with all farms in the sample operating in the same year. We may observe that the wind farms under analysis are more homogenous in 2010 than in 2011 which is confirmed by bootstrapping analysis. The absolute value of bias is slightly higher in 2011 due to the differences between bias-corrected efficiency scores, and efficiency estimates are higher in the same period. Globally, this indicates that farms moved farther from the frontier. This effect is captured by the analysis of efficiency change index (IE) for each farm, which is explored in productivity analysis. Figure 1 presents the average of bias-corrected efficiency of the farms located in the same region for both years which indicates that the level of efficiency spread increased in 2011 for all regions.

\subsection{Benchmarking Analysis}

The benchmark farms and their best practices should be identified in order to be emulated by inefficient units. These practices may be related to the use of more efficient wind turbines, enhanced wind farm design and layout, better operation and maintenance schemes, which may be used to support the inefficient farms to achieve 


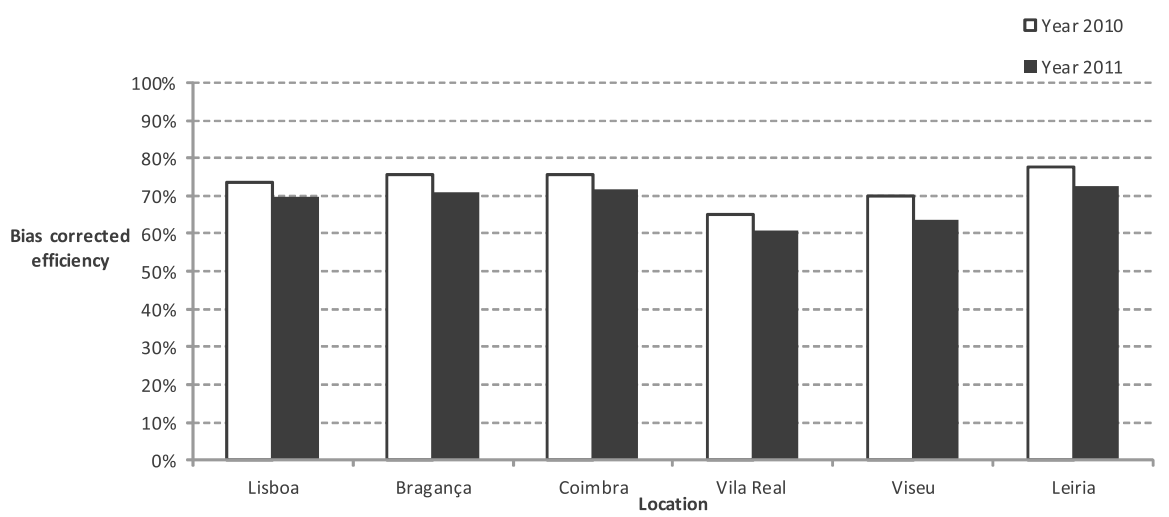

Fig. 1 Comparison of the average of bias-corrected efficiency of the farms located in the same region for 2010 and 2011 years

the appropriate targets. It is also important to identify the nature of returns to scale of the Pareto-efficient farms to explore changes in their size.

From the sample used, there are only 3 efficient farms: Achada, Candeeiros and Pampilhosa. These farms maintain the efficiency status in both years. In 2011, Achada and Candeeiros are the benchmarks, being used as reference 27 and 22 times, respectively. There are no units which are compared with Pampilhosa, since this farm is the largest unit in terms of number of wind turbines and installed capacity. In the following, we explore the profile of the benchmarks in terms of location and type of wind turbines used.

Benchmarks are located in areas with high wind potential (Lisboa, Leiria and Coimbra) and their energy conversion system is based on asynchronous generators. The wind turbines of Achada are from Nordex manufacturer while the wind turbines of Candeeiros and Pampilhosa are from Vestas. These farms are the largest ones while Achada is a smaller farm. Figure 2 compares the age, inputs and output of benchmarks with those observed in inefficient farms, in 2011 (the some profile occurs in 2010). In this graph the scores were normalized by the average scores observed in benchmarks to simplify the comparison. The installed capacity of inefficient units is, on average, $80 \%$ less of that observed in benchmarks and the electric energy generated follows a decrement of the same magnitude. The inefficient units have, on average, $67 \%$ less number of wind turbines of those observed in benchmarks. Given that wind hours in geographical areas where inefficient units are located have a small reduction (about 14\%), this suggests inefficient farms are prone to an overpowering process, in order to increase their output, as they are not exploring all wind energy potential. The fact that inefficient farms are, on average, $15 \%$ older than benchmarks, may explain some inefficiency in some farms.

In both years, the most inefficient unit is the same farm: the Lomba Seixa I with scores equal to $56.7 \%$, in 2010, and $49.4 \%$, in 2011 . The lowest score can be due 


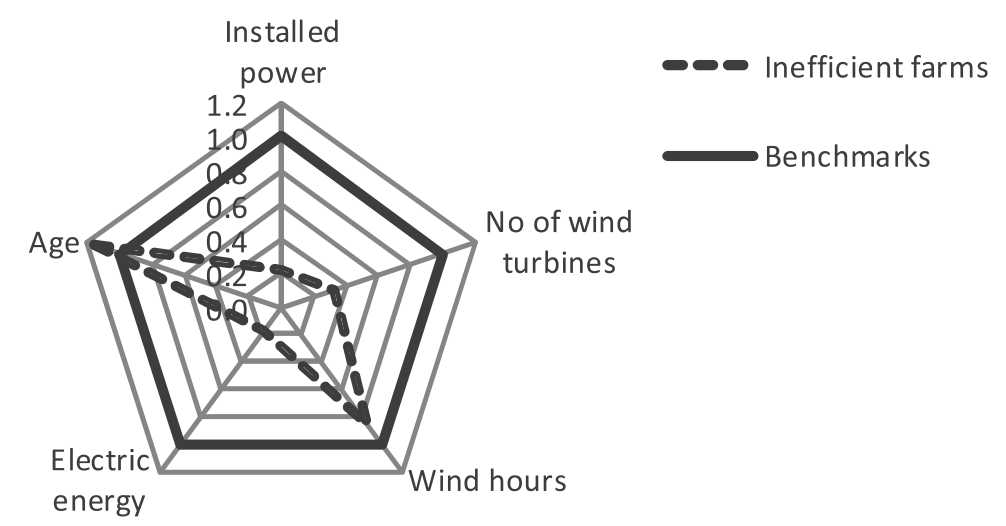

Fig. 2 Comparison between benchmarks and inefficient farms in 2011

to age of technology of the energy conversion system installed in this farm, as it is 11 years old.

The results also indicate that all inefficient farms have slack in the constraint relative to number of wind turbines. Conversely, there are no farms with slack in installed capacity. Thus, the inefficient farms would increase the energy generated by using a lower number of wind turbines with higher rated power, providing the same installed power. This upshot is important in repowering processes. Although, it is important to use a relevant number of turbines to catch the wind potential in a given location, these results suggest that wind farm design could be enhanced and used to decrease the environment impact of future wind farms projects.

These findings should be explored and discussed with the promoter, in order to enhance performance of the wind farms. For inefficient units, it is possible to specify appropriate targets based on internal benchmarking, as proposed in the next section.

\subsubsection{Target setting}

For each inefficient farm, we can define targets for performance improvement. These targets are determined by linear combination of the benchmarks for each inefficient unit. For example, the technical efficiency of farm 17 (Lousã I) is about $67 \%$. The scores for 2011 period, regarding inputs and output of this farm, DEA targets (determined by (2)) and peers, are presented in Table 3 .

The target for a given variable (input or output) of farm 17 is defined by the linear combination of 0.213 of the score observed in farm 1 (Achada) and 0.302 of the score observed in farm 7 (Candeeiros). Farm 17 is larger than farm 1 and smaller than farm 7. Targets indicate that it is possible to increase the electric energy to $107.1 \mathrm{GWh}$ by using the actual installed capacity with the same wind hours available in 2011, with a slack roughly equal to 2 turbines. In theory, the ratio between the total installed power and the number of turbines should be increased, 
Table 3 Target setting of Farm 17

\begin{tabular}{l|l|l|l|l}
\hline & & & Benchmarks \\
\cline { 4 - 5 } & & & Farm 1 & Farm 7 \\
\hline & Observed & Target & $\lambda=0.213$ & $\lambda=0.302$ \\
\hline Installed power $(\mathrm{MW})$ & 35 & 35 & 6.9 & 111 \\
\hline No of wind turbines & 14 & 11.8 & 3 & 37 \\
\hline Wind hours & 2598 & 2598 & 5397 & 4787 \\
\hline Electric energy generated $(\mathrm{GWh})$ & 71.7 & 107.1 & 22.4 & 338.9 \\
\hline
\end{tabular}

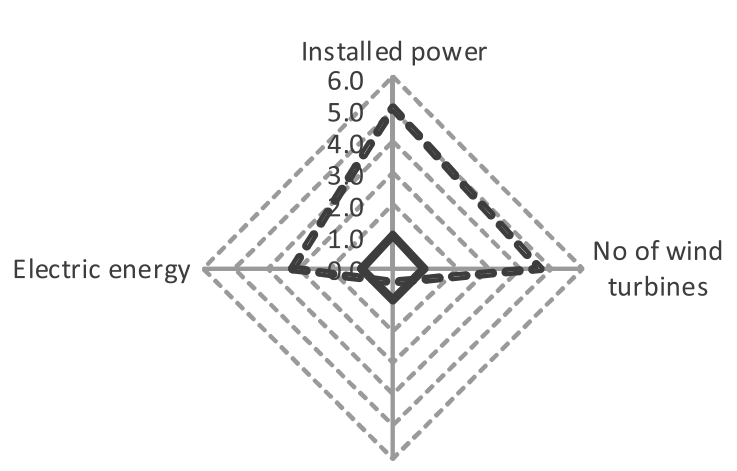

Wind hours

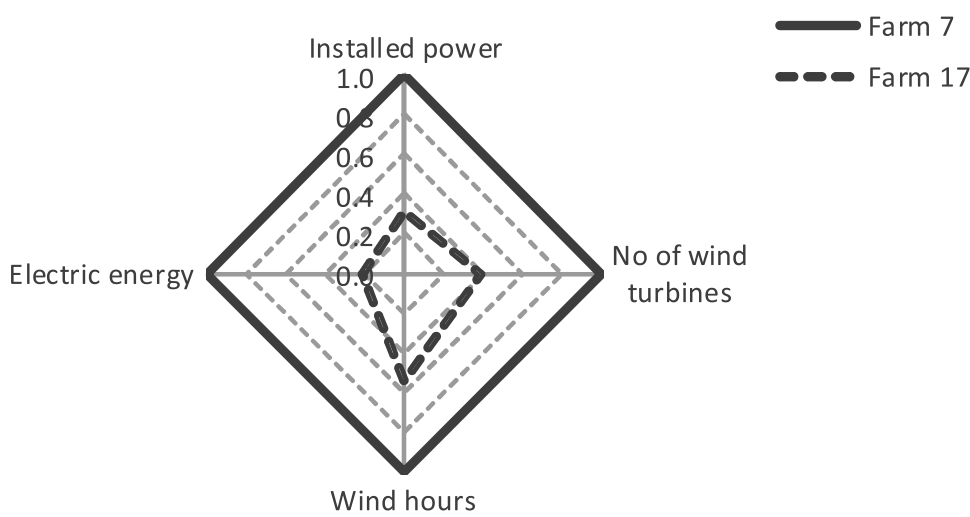

Fig. 3 Comparison between actual values of Farm 17 with benchmarks 1 and 7

via increment of the rated power of each turbine. We can compare the actual inputs and output observed in farm 17 with each benchmark by using the radar graphs in Fig. 3, where the scores were normalized by those observed in benchmarks to simplify the comparison. Farm 17 has an installed power and a number of wind turbines which are almost 5 times higher, but $48 \%$ less wind hours than those observed in benchmark 1. Taking into consideration the exogenous characteristic 
of the input wind hours, it is not evident a possible increase in electric energy production. On the other hand, farm 17 has an installed power and a number of wind turbines about $70 \%$ and $60 \%$, respectively, less than those observed in benchmark 7 while the reduction in the wind hours is about $50 \%$. We can conclude that inputs of farm 17 are, on average, $60 \%$ lower than those observed in benchmark 7 and a similar percentage of reduction in generated electric energy would be expected and not a decrease of $80 \%$, as observed. From the comparison with peers, namely wind farm 7 , the farm 17 could produce higher level of electric energy from the resources observed. Hence, it is necessary to identify the best practices observed in benchmarks 1 and 7 which should be emulated by inefficient farm 17 .

As the production technology of wind farms is characterized by constant returns to scale, the farm efficiency score, $1 / \theta^{*}$, includes sources related to the inefficient operation and scale size. Next, we explore the scale size of the farms based on internal benchmarking.

\subsubsection{Exploring Changes in Wind Farms Size}

As the scale size affects the productivity of a DMU, it is important to calculate the scale efficiency to measure the distance between CRS and VRS frontiers at the scale size of the assessed unit. So, the larger the difference between TE and PTE efficiency scores, the lower the value of scale efficiency is, and the adverse impact of scale size on productivity is more significative. The average scale efficiency score is, on average, $95.38 \%$, and $93.31 \%$, in 2010 and 2011, respectively. This means that scale size only affects the productivity of a small proportion of units observed (Jarmeleira, Borninhos, Rabaçal, Chiqueiro, Malhadizes, Degracias, Lousã I, Lousã II, Malhadas), where the scale efficiency has the lowest scores, with a range between $73.4 \%$ and $88.9 \%$. This strengthens the use of constant returns to scale frontier technology to assess the wind farms efficiency.

The analysis of local returns to scale according to [8] shows that Achada, Candeeiros and Pampilhosa are characterized by an optimum size. Jarmeleira, Lousã II, Malhadas and Rabaçal have increasing returns to scale, which indicates that the size of these units could be increased with a repowering process which enables increasing their productivity. There is no unit which has decreasing returns to scale and, consequently, there is no one with higher size than the required, taken into account the level of electric energy generated.

\subsection{Productivity Analysis}

In a second stage, we investigate the productivity of wind farms by disentangling the efficiency change and technological change effects observed in wind farms in 2010 and 2011. An aggregate analysis is performed by identifying the global effects which had occurred in the period under analysis. Changes in efficiency 
Table 4 Significant scores for $I, I E$ and $I F$

\begin{tabular}{l|r|r|l}
\hline & \multicolumn{1}{|l|}{ I } & \multicolumn{1}{l|}{ IE } & \multicolumn{1}{c}{ IF } \\
\hline Improvement & 1 & 1 & - \\
\hline Deterioration & 27 & 19 & 17 \\
\hline Stagnation & 2 & 10 & 13 \\
\hline
\end{tabular}

$(I E)$, technology $(I F)$ and productivity $(I)$ indexes of farms are explored through identification of scores higher, lower or equal to 1 which correspond to improvement, deterioration or stagnation, respectively. This analysis is complemented with bootstrapping framework, as proposed by [18], to identify if those changes, for each farm, are significant. Table 4 aggregates the significant results in terms of number of wind farms which improve, decline or maintain the performance for each index.

We observed that 27 farms decreased overall productivity levels in year 2011, as indicated by significant scores of $I$ index. This effect is mainly due to deterioration in the productivity levels of the frontier for some inputs-output mix and decreasing efficiency levels in some farms. Only Serra Escusa improves overall productivity level due to improvement on its efficiency in 2011. Pampilhosa and Candeeiros maintain overall productivity levels in 2011.

There are 19 farms that moved farther from the frontier in 2011, as indicated by significant scores of $I E$ index. These farms had the worst performance in 2011, so the reasons for that should be investigated. Only Serra Escusa moved closer to the best practices. It is recommended to identify how this farm carried out its operations and maintenance services in order to be emulated by the inefficient farms. The remaining farms maintained the efficiency spread levels observed in 2010.

Globally, the productivity of the best-practices frontier decreased considerably in 2011 for the input-output combinations of 17 farms, although for the remaining input-output mix, the frontier maintained the level of productivity observed in 2010. This is connected with the reduction of electric energy generated observed in wind energy sector in 2011. Next, we explore the relative position of frontiers for the farms observed in each period. Thus, we analyze if the ratios of $I F\left(\frac{E^{2010}(2011)}{E^{2011}(2011)}\right.$, $\left.\frac{E^{2010}(2010)}{E^{2011}(2010)}\right)$ are statistical significant throughout bootstrap framework [18]. Table 5 aggregates the significant results in terms of number of wind farms which improve, decline or maintain the performance for each ratio.

The inputs-output combinations of 14 farms observed in 2010 are located in areas of the PPS where the productivity of the frontier declined. The remaining farms are located in areas of the PPS where the frontier maintained the productivity. During 2011, there are 18 inputs-output combinations of wind farms located in areas of the PPS where the frontier regressed, while the other remaining farms are located in areas where the frontier maintained the productivity. There is no statistical evidence of crossed frontiers for all input-output combinations. 
Table 5 Significant scores for ratios of $I F$

\begin{tabular}{l|l|l}
\hline & $E^{2010}(2010) / E^{2011}(2010)$ & $E^{2010}(2011) / E^{2011}(2011)$ \\
\hline Improvement & 0 & 0 \\
\hline Deterioration & 14 & 18 \\
\hline Stagnation & 16 & 12 \\
\hline
\end{tabular}

\section{Conclusions}

This study proposes a methodology to assess the efficiency and productivity change of wind farms, which can support decision makers during operating phase of wind farms, in repowering processes and also in project design and layout of new farms. In a first stage, the efficiency assessment of wind farms enables the identification of benchmark profiles, setting targets for inefficient units and also the exploitation of the scale size of existing farms. The second stage explores the efficiency and productivity over time of wind farms by identifying the global effects which occurred in terms of changes in internal practices observed and productivity of the frontier, during the period under analysis. These findings correspond to the additional insights regarding the efficiency and productivity assessment of wind farms which make this paper different from the previous studies.

Regarding the operating stage of the farms analyzed, 3 farms are the benchmarks, whose best practices can be related to the well-performing operations and maintenance programs. Between 2010 and 2011, different profiles of wind farms were identified in terms of overall productivity change, efficiency change and technological change. Almost all farms decreased overall productivity levels, mainly due to the decline in the productivity levels of the frontier, which is in accordance with the decrease in wind availability, measured in wind hours, observed in 2011. The productivity of the frontier declined for some input-output combinations observed in 2011 and for the other combinations, the frontier maintained its productivity. In the later case, there is one farm that improved its overall productivity due to the improvement of its efficiency in 2011 and two farms which maintained overall productivity as they kept the efficiency levels. We observed also that 19 farms had the worst performance in 2011 which requires further investigation to reveal the reasons.

Further research should be conducted using a larger panel data set in order to analyze the impact of wind availability on the productivity of wind farms. The inclusion of variables concerning the operation and maintenance schemes should also be explored in future performance assessments of wind farms. 


\section{References}

1. Banker, R.D., Charnes, A., Cooper, W.W.: Some models for estimating technical and scale inefficiencies in Data Envelopment Analysis. Manag. Sci. 30(9), 1078-1092 (1984)

2. Camanho, A.S., Portela, M.C., Vaz, C.B.: Efficiency analysis accounting for internal and external non-discretionary factors. Comput. Oper. Res. 36(5), 1591-1601 (2009)

3. Caves, D.W., Christensen, L.R., Diewert, W.E.: The economic theory of index numbers and the measurement of input, output and productivity. Econometrica 50, 1393-1414 (1982)

4. Charnes, A., Cooper, W.W., Rhodes, E.: Measuring efficiency of decision-making units. Eur. J. Oper. Res. 2(6), 429-444 (1978)

5. Dyson, R.G., Allen, R., Camanho, A.S., Podinovski, V.V., Sarrico, C.S., Shale, E. A.: Pitfalls and protocols in DEA. Eur. J. Oper. Res. 132, 245-259 (2001)

6. Direção Geral de Energia e Geologia (DGEG): Renováveis, Estatísticas rápidas (Nov. 2013). Available from: http://www.dgeg.pt/

7. Efron, B.: Bootstrap method: another look at the jackknife. Ann. Stat. 7(1), 1-26 (1979)

8. Färe, R., Grosskopf, S., Lovell, C.A.K.: The Measurement of Efficiency of Production. Kluwer, Boston (1985)

9. Färe, R., Grosskopf, S., Lindgren, B., Roos, P.: Productivity developments in swedish hospitals: a Malmquist output index approach. In: Charnes, A., Cooper, W.W., Lewin, A., Seiford, L. (eds.) Data Envelopment Analysis: Theory, Methodology and Applications, pp. 253-272. Kluwer, Boston (1994)

10. Gilbert, R.A., Wilson, P.W.: Effects of deregulation on the productivity of Korean banks. J. Econ. Bus. 50(2), 133-155 (1998)

11. Horta, I.M., Camanho, A.S., Johnes, J., Johnes, G.: Performance trends in the construction industry worldwide: an overview of the turn of the century. J. Product. Anal. 39(1), 89-99 (2012)

12. Iglesias, G., Castellanos, P., Seijas, A.: Measurement of productive efficiency with frontier methods: a case study for wind farms. Energy Econ. 32(5), 1199-1208 (2010)

13. International Energy Agency (IEA): World Energy Outlook 2010. IEA Publications, Paris (2010)

14. Martins, A.C., Marques, R.C., Cruz, C.O.: Public-private partnerships for wind power generation: the Portuguese case. Energy Policy 39, 94-104 (2011)

15. Odeck, J.: Statistical precision of DEA and malmquist indices: a bootstrap application to norwegian grain producers. Omega 37(5), 1007-1017 (2009)

16. Pestana Barros, C., Sequeira Antunes, O.: Performance assessment of portuguese wind farms: ownership and managerial efficiency. Energy Policy 39(6), 3055-3063 (2011)

17. Simar, L., Wilson, P.W.: Sensitivity analysis of efficiency scores: how to bootstrap in nonparametric frontier models. Manag. Sci. 44(1), 49-61 (1998)

18. Simar, L., Wilson, P.W.: Estimating and bootstrapping Malmquist indices. Eur. J. Oper. Res. 115, 459-471 (1999)

19. Tortosaausina, E., Grifelltatje, E., Armero, C., Conesa, D.: Sensitivity analysis of efficiency and malmquist productivity indices: an application to spanish savings banks. Eur. J. Oper. Res. 184(3), 1062-1084 (2008)

20. Wilson P.W.: FEAR: a software package for frontier efficiency analysis with R. Socio-Econ. Plan. Sci. 42(4), 247-254 (2008)

21. Zhou, P., Ang, B.W., Poh, K.L.: A survey of data envelopment analysis in energy and environmental studies. Eur. J. Oper. Res. 189(1), 1-18 (2008) 